\title{
Angewandte
}

Supporting Information

(c) Wiley-VCH 2006

69451 Weinheim, Germany 


\section{Angewandte Chemie}

\section{Multifunctional Iridium Complexes Based on Carbazole Modules as Highly Efficient Electrophosphors}

Wai-Yeung Wong, * Cheuk-Lam Ho, Zhi-Qiang Gao, Bao-Xiu Mi, Chin-Hsin Chen, Kok-Wai Cheah, and Zhenyang Lin 


\section{Experimental Section}

General Information: All reactions were performed under nitrogen. Solvents were carefully dried and distilled from appropriate drying agents prior to use. Commercially available reagents were used without further purification unless otherwise stated. All reactions were monitored by thin-layer chromatography (TLC) with Merck pre-coated glass plates. Flash column chromatography and preparative TLC were carried out using silica gel from Merck (230-400 mesh). Fast atom bombardment (FAB) mass spectra were recorded on a Finnigan MAT SSQ710 system. Proton NMR spectra were measured in $\mathrm{CDCl}_{3}$ on a Varian Inova $400 \mathrm{MHz}$ FT-NMR spectrometer; chemical shifts were quoted relative to tetramethylsilane.

Physical Measurements: UV-vis spectra were obtained on a HP-8453 spectrophotometer. The photoluminescent properties and lifetimes of the compounds were probed on the Photon Technology International (PTI) Fluorescence Master Series QM1 system. The phosphorescence quantum yields were determined in $\mathrm{CH}_{2} \mathrm{Cl}_{2}$ solutions at $293 \mathrm{~K}$ against $f a c$-[ $\left.\operatorname{Ir}(\mathrm{ppy})_{3}\right]$ as a reference standard $\left(\Phi_{\mathrm{P}}=0.40\right)$. For solid-state emission spectral measurements, the $325 \mathrm{~nm}$ line of a He-Cd laser was used as an excitation source. The luminescence spectra were analyzed by a $0.25 \mathrm{~m}$ focal length double monochromator with a Peltier cooled photomultiplier tube and processed with a lock-in-amplifier. Electrochemical measurements were made using a BAS CV-50W model potentiostat. A conventional three-electrode configuration consisting of a platinum working electrode, a Pt-wire counter electrode, and an $\mathrm{Ag} / \mathrm{AgCl}$ reference electrode was used. The supporting electrolyte was $0.1 \mathrm{M}\left[\mathrm{Bu}_{4} \mathrm{~N} \mathrm{PF}_{6}\right.$. Ferrocene was added as a calibrant after each set of measurements, and all potentials reported were quoted with reference to the ferrocene-ferrocenium $\left(\mathrm{Fc} / \mathrm{Fc}^{+}\right)$couple at a scan rate of $100 \mathrm{mV} \mathrm{s}^{-1}$. Thermal analyses were performed with the Perkin-Elmer TGA6 thermal analyzer. 
Preparation of 1 and 2: First, the $N$-arylation of carbazole was achieved in a large scale by the Ullmann-type condensation between the easily accessible carbazole and $p$-iodoarene. ${ }^{[1]}$ After bromination with one equivalent of $N$-bromosuccimide (NBS) to give the $N$-arylated 3bromocarbazoles, the carbazolylpyridine ligands $\mathbf{L}_{\mathbf{1}}$ and $\mathbf{L}_{\mathbf{2}}$ were prepared by reacting 3-bromo-9arylcarbazole $(6.21 \mathrm{mmol})$ with 1.2 equivalent of 2-(tributylstannyl)pyridine $(7.45 \mathrm{mmol})$ in toluene (30 $\mathrm{mL})$ in the presence of $\left[\mathrm{Pd}\left(\mathrm{PPh}_{3}\right)_{4}\right](0.07 \mathrm{~g})$ under nitrogen. After reflux for 2 days, the dark mixture was poured into water and extracted with ethyl acetate. The concentrated filtrate was purified on a silica column to give $\mathbf{L}_{\mathbf{1}}$ and $\mathbf{L}_{2}$ as brown oils in 54-60\%. [ $\operatorname{Ir}(\mathrm{acac})_{3}$ ] $(0.30 \mathrm{mmol})$ and an excess of $\mathbf{L}_{\mathbf{1}}$ (or $\mathbf{L}_{\mathbf{2}}$ ) (1.04 mmol) were heated to reflux in glycerol $(10 \mathrm{~mL})$ for $24 \mathrm{~h}$. The solution was then cooled and extracted with $\mathrm{CH}_{2} \mathrm{Cl}_{2}$. The concentrated filtrate was purified by TLC over silica using $\mathrm{CH}_{2} \mathrm{Cl}_{2}$ as eluent. From the first bright yellow band in each case, $\mathbf{1}$ and $\mathbf{2}$ can be obtained as yellow solids. Spectral Data: 1: 35\%. ${ }^{1} \mathrm{H}$ NMR $\left(\mathrm{CDCl}_{3}\right): \delta(\mathrm{ppm}) 8.29$ (s, 3H, Ar), 8.00 (m, 6H, Ar), 7.76 (m, 3H, Ar), 7.57 (m, 3H, Ar), 7.32-7.25 (m, 9H, Ar), 6.90 (m, 9H, Ar), 6.62 (m, 9H, Ar), 6.38 (m, 3H, Ar). FAB-MS (m/z): $1150\left[\mathrm{M}^{+}\right]$. Anal. calcd. for $\mathrm{C}_{69} \mathrm{H}_{45} \mathrm{~N}_{6} \mathrm{Ir}: \mathrm{C}, 72.04 ; \mathrm{H}, 3.94 ; \mathrm{N}, 7.31$; found: C, 71.89; H, 3.76; N, 7.10. 2: 53\%. ${ }^{1} \mathrm{H}$ NMR $\left(\mathrm{CDCl}_{3}\right): \delta(\mathrm{ppm}) 8.34$ (s, 3H, Ar), 8.04-8.02 (d, $\left.J=6.8 \mathrm{~Hz}, 6 \mathrm{H}, \mathrm{Ar}\right)$, 7.66-7.61 (m, 6H, Ar), 7.28-7.22 (m, 9H, Ar), 6.87-6.82 (m, 9H, Ar), 6.70 (s, 3H, Ar), 6.33-6.27 (t, J $=8.9 \mathrm{~Hz}, 6 \mathrm{H}, \mathrm{Ar})$. FAB-MS (m/z): $1204\left[\mathrm{M}^{+}\right]$. Anal. calcd. for $\mathrm{C}_{69} \mathrm{H}_{42} \mathrm{~N}_{6} \mathrm{~F}_{3} \mathrm{Ir}: \mathrm{C}, 68.81 ; \mathrm{H}, 3.52 ; \mathrm{N}$, 6.98; found: C, 68.65; H, 3.44; N, 6.64 . 

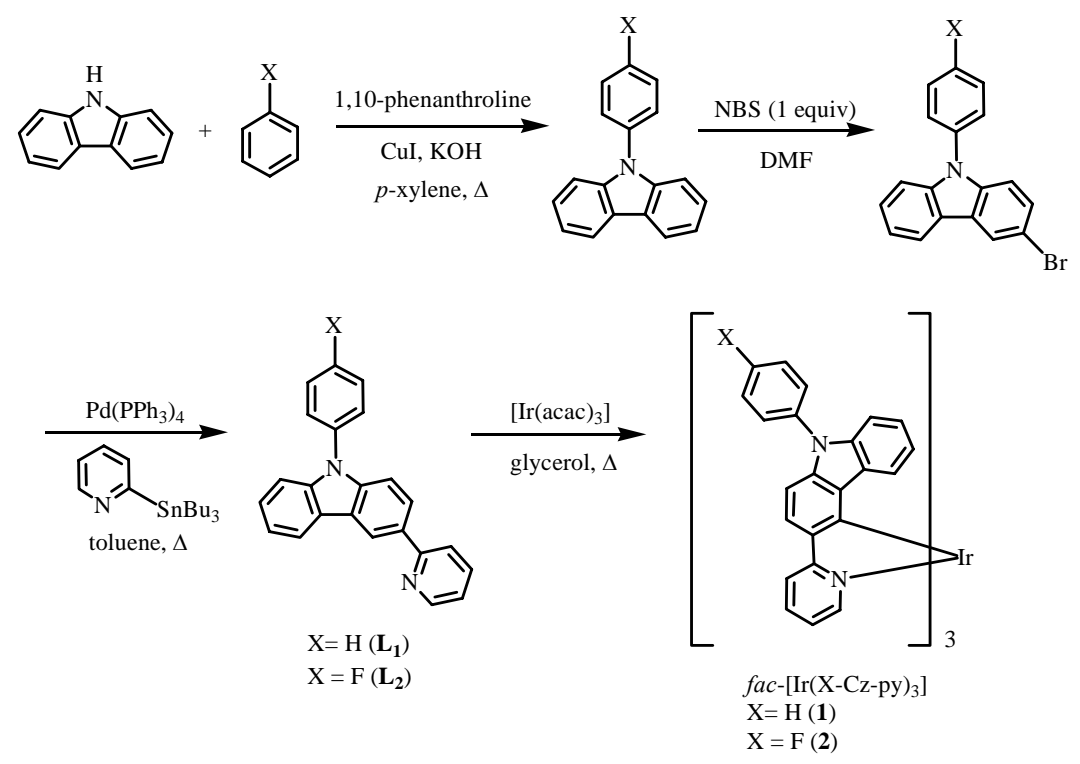

Scheme 1. Synthesis of new homoleptic iridium(III) complexes $f a c-[\operatorname{Ir}(\mathrm{X}-\mathrm{Cz}-\mathrm{py})]_{3} \mathbf{1}$ and $\mathbf{2}$.

X-ray Crystallographic Details. Yellow crystals of $\mathbf{1}$ suitable for X-ray diffraction studies were grown by slow evaporation of its solution in $\mathrm{CH}_{2} \mathrm{Cl}_{2}$ /diethyl ether at room temperature. Geometric and intensity data were collected at $293 \mathrm{~K}$ using graphite-monochromated Mo-K $\alpha$ radiation $(\lambda=0.71073$ $\AA$ ) on a Bruker Axs SMART 1000 CCD diffractometer. The collected frames were processed with the software SAINT ${ }^{[2 a]}$ and an absorption correction (SADABS) ${ }^{[2 b]}$ was applied to the collected reflections. The structure was solved by the Direct methods (SHELXTL) ${ }^{[3]}$ in conjunction with standard difference Fourier techniques and subsequently refined by full-matrix least-squares analyses on $F^{2}$. Hydrogen atoms were generated in their idealized positions and all non-hydrogen atoms were assigned with anisotropic displacement parameters. Crystal data for 1: $\mathrm{C}_{69} \mathrm{H}_{45} \mathrm{~N}_{6} \mathrm{Ir} \cdot 2 \mathrm{CH}_{2} \mathrm{Cl}_{2}, M_{\mathrm{w}}=1320.16$, monoclinic, space group $P 1, a=12.7149(6), b=14.1335(6), c=17.7369(8) \AA, \alpha=81.235(1), \beta=$ 74.257(1), $\gamma=71.128(1)^{\circ}, V=2895.6(2) \AA^{3}, Z=2, \rho_{\text {calcd }}=1.514 \mathrm{mg} \mathrm{m}^{-3}, \mu\left(\mathrm{Mo}_{\mathrm{K} \alpha}\right)=2.540 \mathrm{~mm}^{-1}$, $F(000)=1324, T=293 \mathrm{~K} .17323$ reflections measured, of which 14895 were unique $\left(R_{\text {int }}=0.0197\right)$. Final $R_{1}=0.0347$ and $w R_{2}=0.0790$ for 12252 observed reflections with $I>2 \sigma(I)$. CCDC-607186 contains the supplementary crystallographic data for this paper. These data can be obtained free of charge from The Cambridge Crystallographic Data Centre via www.ccdc.cam.ac.uk/data_request/cif. 
Molecular Orbital Calculations. Density functional calculations at the B3LYP level were performed based on their experimental geometry obtained from the X-ray data. The basis set used for C, N, O and $\mathrm{H}$ atoms was 6-31G while effective core potentials with a LanL2DZ basis set were employed for the Ir atom. All the calculations were carried out using the Gaussian 03 program. ${ }^{[5]}$ All the MO plots were made with the use of Molden 3.5. ${ }^{[6]}$

\section{References:}

[1] a) J. Hassan, M. Sévignon, C. Gozzi, E. Schulz, M. Lemaire, Chem. Rev. 2002, 102, 1359. b) M. Martínez-Palau, E. Perea, F. López-Calahorra, D. Velasco, Lett. Org. Chem. 2004, 1, 231.

[2] a) SAINT+, ver. 6.02a, Bruker Analytical X-ray System, Inc., Madison, WI, 1998. b) G. M. Sheldrick, SADABS, Empirical Absorption Correction Program; University of Göttingen, Germany, 1997.

[3] G. M. Sheldrick, SHELXTL ${ }^{\mathrm{TM}}$, Reference manual, ver. 5.1, Madison, WI, 1997.

[4] a) W. R. Wadt, P. J. Hay, J. Chem. Phys. 1985, 82, 284-298; b) P. J. Hay, W. R. Wadt, J. Chem. Phys. 1985, 82, 299-310.

[5] M. J. Frisch, G. W. Trucks, H. B. Schlegel, G. E. Scuseria, M. A. Robb, J. R. Cheeseman, J. A. Montgomery, T. Vreven, Jr., K. N. Kudin, J. C. Burant, J. M. Millam, S. S. Iyengar, J. Tomasi, V. Barone, B. Mennucci, M. Cossi, G. Scalmani, N. Rega, G. A. Petersson, H. Nakatsuji, M. Hada, M. Ehara, K. Toyota, R. Fukuda, J. Hasegawa, M. Ishida, T. Nakajima, Y. Honda, O. Kitao, H. Nakai, M. Klene, X. Li, J. E. Knox, H. P. Hratchian, J. B. Cross, C. Adamo, J. Jaramillo, R. Gomperts, R. E. Stratmann, O. Yazyev, A. J. Austin, R. Cammi, C. Pomelli, J. W. Ochterski, P. Y. Ayala, K. Morokuma, G. A. Voth, P. Salvador, J. J. Dannenberg, V. G. Zakrzewski, S. Dapprich, A. D. Daniels, M. C. Strain, O. Farkas, D. K. Malick, A. D. Rabuck, K. Raghavachari, J. B. Foresman, J. V. Ortiz, Q. Cui, A. G. Baboul, S. Clifford, J. Cioslowski, B. B. Stefanov, G. Liu, A. Liashenko, P. Piskorz, I. Komaromi, R. L. Martin, D. J. Fox, T. 
Keith, M. A. Al-Laham, C. Y. Peng, A. Nanayakkara, M. Challacombe, P. M. W. Gill, B. Johnson, W. Chen, M. W. Wong, C. Gonzalez, J. A. Pople, Gaussian 03, revision B05, Gaussian, Inc., Pittsburgh, PA, 2003.

[6] G. Schaftenaar, Molden v3.5, CAOS/CAMM Center Nijmegen, Toernooiveld, Nijmegen, Netherlands, 1999.

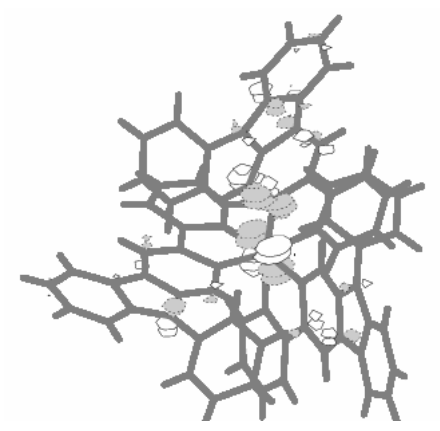

HOMO-1 (-0.17056 au)

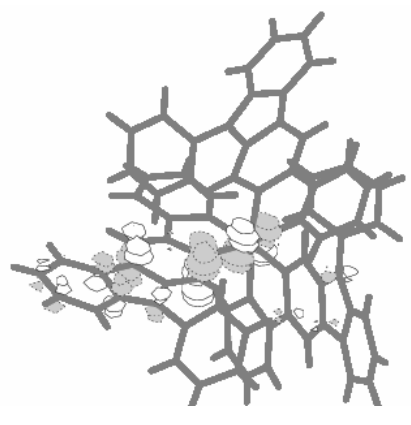

HOMO (-0.16412 au)

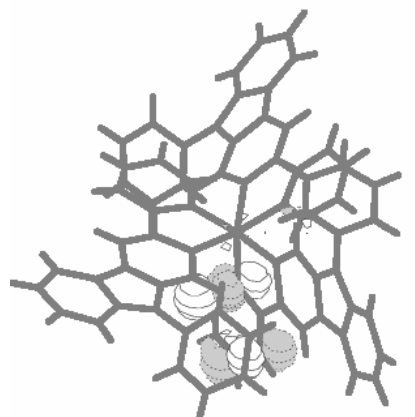

LUMO (-0.04376 au)

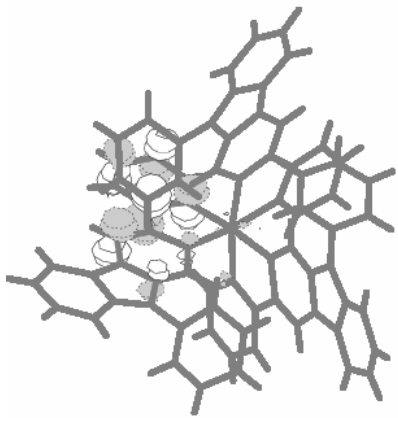

LUMO+1 (-0.04246 au)

Figure S1. Contour plots of the highest occupied (HOMO) and lowest unoccupied (LUMO) molecular orbitals for $\mathbf{1}$ as determined by the DFT calculations. $(1 \mathrm{au}=27.2114 \mathrm{eV})$

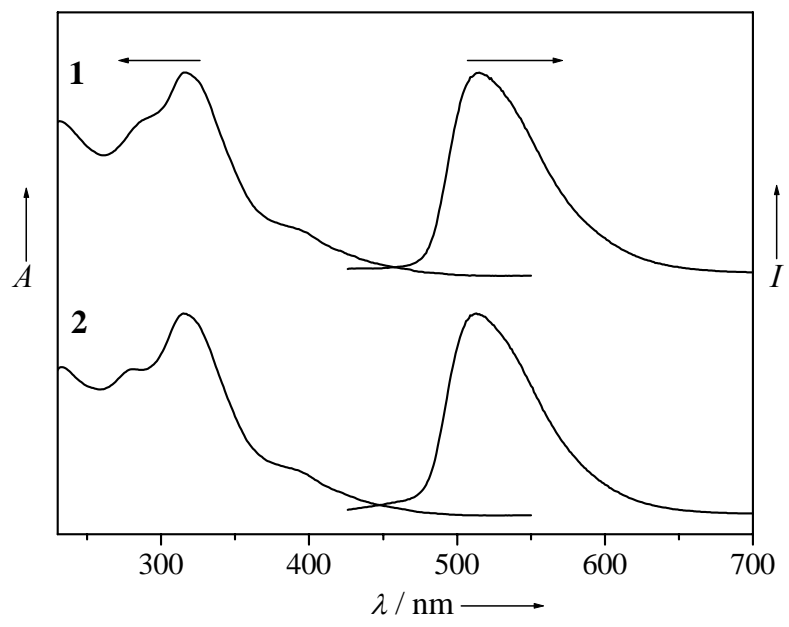

Figure S2. Absorption and PL spectra of $\mathbf{1}$ and 2 in $\mathrm{CH}_{2} \mathrm{Cl}_{2}$ at $293 \mathrm{~K}$. 


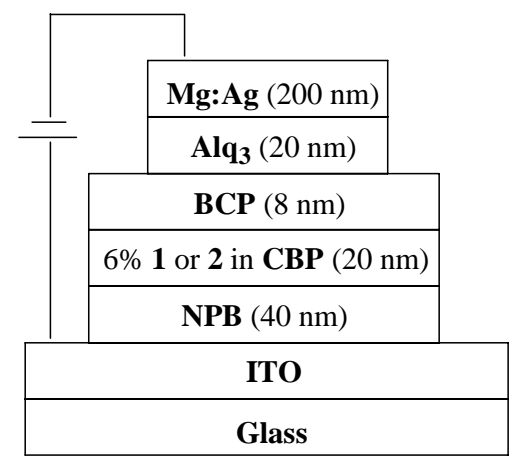

$\begin{array}{cc}\text { Device } & \text { Dopant } \\ \text { I } & \mathbf{1} \\ \text { II } & \mathbf{2}\end{array}$

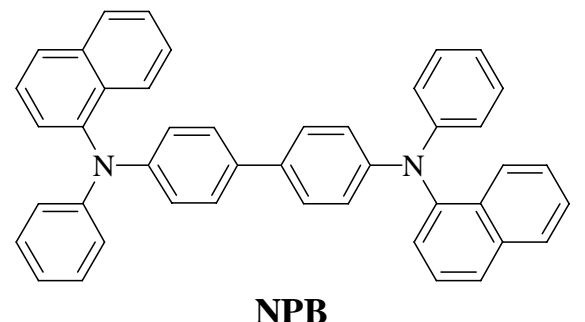

NPB

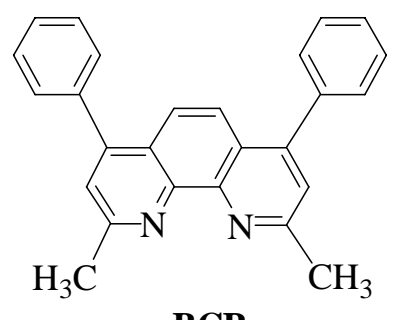

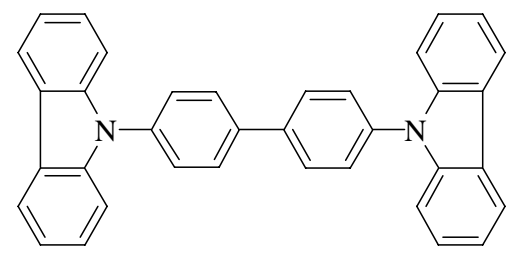

CBP

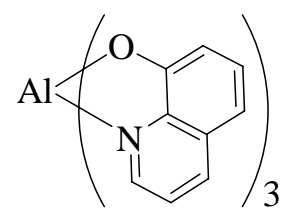

$\mathrm{Alq}_{3}$

\section{BCP}

Figure S3. The general structure for OLED devices and the molecular structures of the compounds used in these devices.

Procedures of Device Fabrication. All OLEDs with the emission area of $0.1 \mathrm{~cm}^{2}$ were fabricated on the pre-patterned ITO-coated glass substrate having an ITO sheet resistance of $30 \Omega /$ sq. Substrates were cleaned by ultrasonication in detergent and dried at $120^{\circ} \mathrm{C}$ for more than $2 \mathrm{~h}$. The substrates were treated by UV ozone before loaded into the vacuum chamber. The doped emission layer was deposited by co-evaporation from two separate sources. The vacuum was less than $5 \times 10^{-6}$ torr during all organic materials deposition and below $8 \times 10^{-6}$ torr during cathode depositon. The $J-V-L$ characteristics of the devices were measured with a computer controlled KEITHLEY 236 source meter and PHOTORESEARCH PR650 spectrophotometer in air without device encapsulation. All the measurements finished within 20 min after the device was unloaded from the vacuum chamber. 


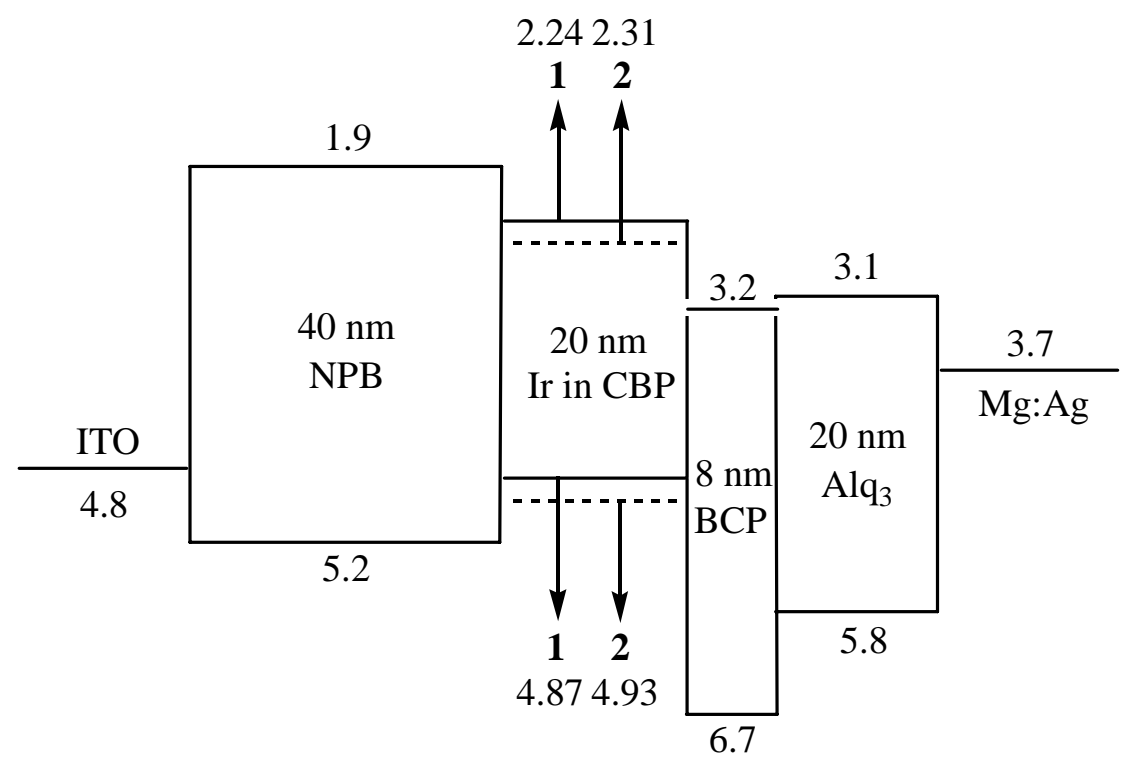

Figure S4. Energy level diagram of the electrophosphorescent devices used in this work. All energy levels are quoted in $\mathrm{eV}$.

Table S1. Performance of Ir-doped electrophosphorescent OLEDs.

\begin{tabular}{ccccccccc}
\hline Device & $\begin{array}{c}\text { Phosphor } \\
\text { dopant }\end{array}$ & $\begin{array}{c}V_{\text {turn-on }} \\
{[\mathrm{V}]}\end{array}$ & $\begin{array}{c}\text { Luminance } \\
{\left[\mathrm{cd} \mathrm{m}^{-2}\right]}\end{array}$ & $\begin{array}{c}\eta_{\text {ext }} \\
{[\%]}\end{array}$ & $\begin{array}{c}\eta_{\mathrm{L}} \\
{\left[\mathrm{cd} \mathrm{A}^{-1}\right]}\end{array}$ & $\begin{array}{c}\eta_{\mathrm{p}} \\
{\left[\mathrm{lm} \mathrm{W}^{-1}\right]}\end{array}$ & $\begin{array}{c}\lambda_{\max } \\
{[\mathrm{nm}][\mathrm{d}]}\end{array}$ & CIE \\
\hline $\mathbf{I}$ & $\mathbf{1}$ & 4.4 & $14730(12)[\mathrm{a}]$ & $11.56(5.2)[\mathrm{a}]$ & $38.01(5.2)[\mathrm{a}]$ & $23.92(4.8)[\mathrm{a}]$ & 508 & $x=0.24$ \\
& & & & $10.62[\mathrm{~b}]$ & $35.19[\mathrm{~b}]$ & $16.96[\mathrm{~b}]$ & $(56)$ & $y=0.63$ \\
& & & & $8.32[\mathrm{c}]$ & $27.47[\mathrm{c}]$ & $10.33[\mathrm{c}]$ & & \\
II & 2 & 4.8 & \multirow{2}{*}{$19360(12)[\mathrm{a}]$} & $6.69(5.6)[\mathrm{a}]$ & $21.35(5.6)[\mathrm{a}]$ & $12.33(5.2)[\mathrm{a}]$ & 508 & $x=0.27$ \\
& & & & $6.22[\mathrm{~b}]$ & $20.02[\mathrm{~b}]$ & $9.66[\mathrm{~b}]$ & $(80)$ & $y=0.60$ \\
& & & & $5.16[\mathrm{c}]$ & $16.63[\mathrm{c}]$ & $6.60[\mathrm{c}]$ & & \\
\hline
\end{tabular}

[a] Maximum values of the devices. Values in parentheses are the voltages at which they were obtained. [b] At $100 \mathrm{~cd} \mathrm{~m}^{-2}$. [c] At $1000 \mathrm{~cd} \mathrm{~m}^{-2}$. [d] fwhm [nm] are shown in parentheses. 

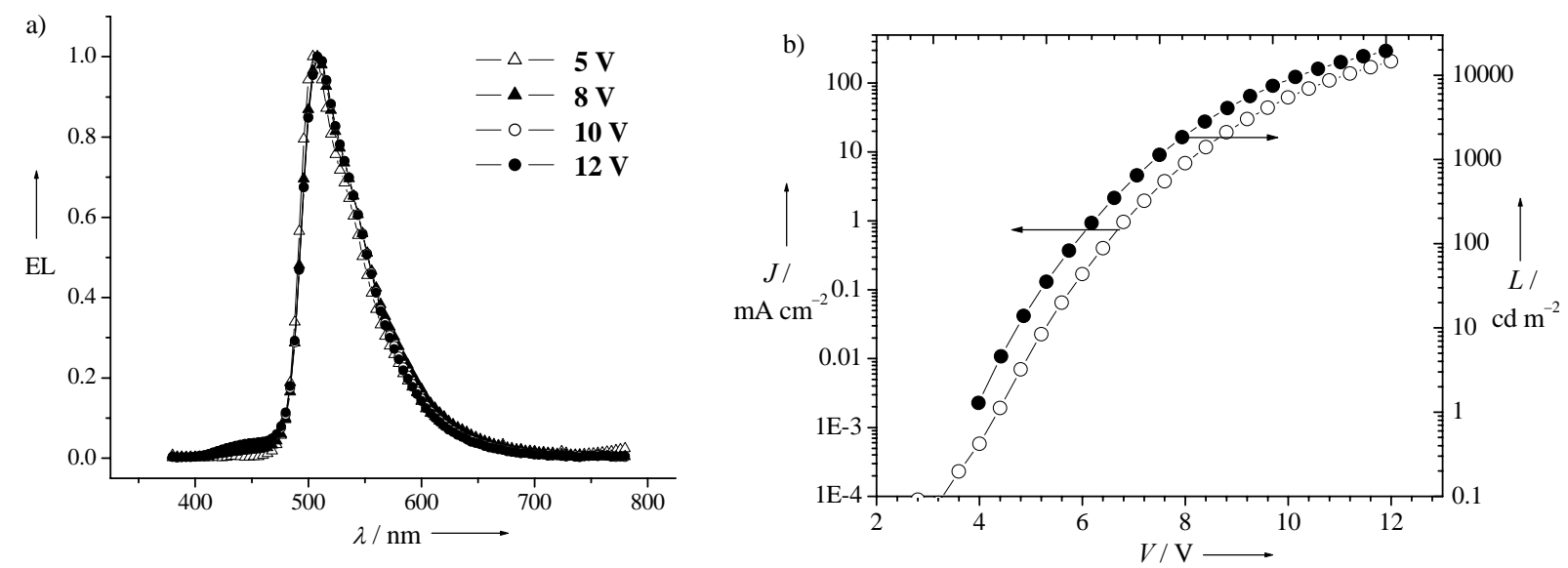

Figure S5. (a) The EL spectra at various voltages and (b) current-voltage-luminance $(J-V-L)$ characteristics for device II.
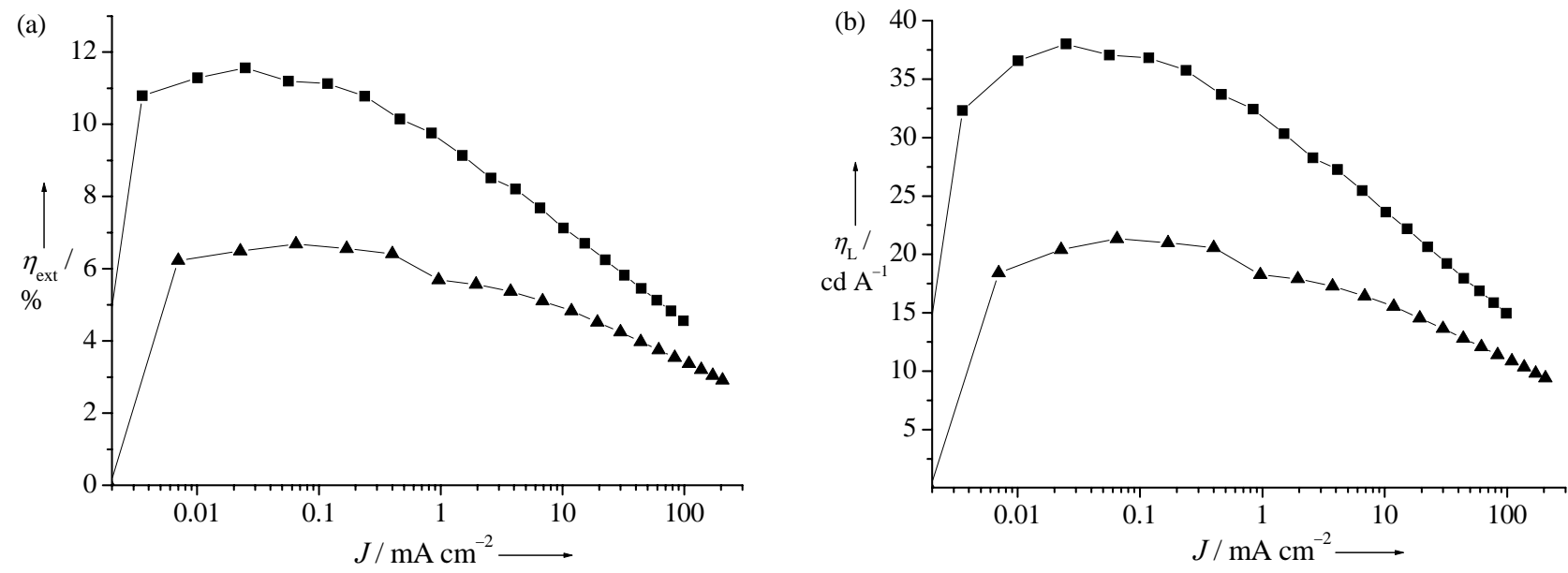

Figure S6. (c) External quantum efficiency versus current density curves for devices I ( $\boldsymbol{\square})$ and II (A) and (d) luminance efficiency versus current density curves for devices I ( $\boldsymbol{\square})$ and II (A). 